\title{
Tests d'évaluation du degré de pollution des sédiments marins : effets sur la production de larves et la consommation d'algues chez le copépode Tigriopus brevicornis \\ Evaluation of the degree of pollution of marine sediments by means of two sublethal tests applied on the copepod Tigriopus brevicornis Effects on larval production and the consumption of algae
}

\author{
J. C. Lacaze et F. Paquet
}

Volume 2, numéro 1, 1989

URI : https://id.erudit.org/iderudit/705020ar

DOI : https://doi.org/10.7202/705020ar

Aller au sommaire du numéro

Éditeur(s)

Université du Québec - INRS-Eau, Terre et Environnement (INRS-ETE)

ISSN

0992-7158 (imprimé)

1718-8598 (numérique)

Découvrir la revue

Citer cet article

Lacaze, J. C. \& Paquet, F. (1989). Tests d'évaluation du degré de pollution des sédiments marins : effets sur la production de larves et la consommation d'algues chez le copépode Tigriopus brevicornis. Revue des sciences de l'eau / Journal of Water Science, 2(1), 1-12. https://doi.org/10.7202/705020ar
Résumé de l'article

L'eau interstitielle ou l'eau de lessivage d'un sédiment est mise en contact avec des lots de 50 femelles ovigères du copépode Tigriopus brevicornis. Notons que ce microcrustacé marin est sensible à la pollution mais résistant aux caractéristiques physico-chimiques des types d'eaux testés (salinité et température notamment). On évalue ensuite les effets de ces eaux sur la production larvaire en 10 jours et sur l'ingestion en 4 jours par les copépodes d'une suspension de Phaeodactylum tricornutum. Nous observons ici, pour des lots de sédiments provenant de la région de Marseille, des réductions de la production de larves variant de $61 \%$ (Les Embiez) à $90 \%$ (Vaine). Le test "consommation d'algues", bien moins sensible aboutit pour les mêmes stations à des réductions de $19 \%$ à $35 \%$. Le test "production larvaire" du fait de sa plus grande sensibilité doit être préféré au test "consommation d'algues". 


\section{Tests d'évaluation du degré de pollution des sédiments marins : \\ effets sur la production de larves \\ et la consommation d'algues \\ chez le copépode Tigriopus brevicornis}

Evaluation of the degree of pollution

of marine sediments by means of two sublethal tests

applied on the copepod Tigriopus brevicomis

Effects on larval production and the consumption of algae

J.C. LACAZE, F. PAQUET [1]

RÉSUMÉ

L'eau interstitielle ou L'eau de lessivage d'un sédiment est mise en contact avec des lots de 50 femelles ovigères du copépode Tigriopus brevicornis. Notons que ce microcrustacé marin est sensible à la pollution mais résistant aux caractéristiques physico-chimiques des types d'eaux testès (salinité et température notamment). On évalue ensuite les effets de ces eaux sur la production larvaire en 10 jours et sur l'ingestion en 4 jours par les copépodes d'une suspension de Phaeodactylum tricormutum. Nous observons ici, pour des lots de sédiments provenant de la région de Marseille, des réductions de la production de larves variant de $61 \%$ (Les Embiez) a $90 \%$ (Vaine). Le test "consommation d'algues", bien moins sensible aboutit pour les mêmes stations à des réductions de $19 \%$ a $35 \%$. Le test "production larvaire" du fait de sa plus grande sensibilité doit être préféré au test "consommation d'algues".

Mots clés : toxicologie, pollution du sêdiment, copépode, production larvaire, consomation d'algues.

(1) Muséum national d'Histoire naturelle et Institut océanographique, 195 rue Saint-Jacques, 75005 Paris, France. 
Host pollutants discharged into the sea are found in sediments, generally after temporary fixation in planktonic organisms. The slightest discharge leaves a trace in the soft bottoms. Thus, it may be said that these behave as good "data storage indicators" testifying to the degree of pollution present. Sediments therefore represent a privileged field in research on the state of pollution in the aquatic eccsystem. How can the degree of pollution in this field be evaluated?

A chemical analysis of pollutants in sediments is a good means of investigation to detect of degradation in the quality of waters. Most pollutants however are difficult to detect and dose. Moreover, in many cases dosage is tedious and costly. It is from this point of view that biological assays were considered essential.

Interstitial water or water used to wash a sediment was placed in contact with batches of 50 ovigerous females of the copepod Tigriopus brevicormis. This marine microcrustacean is known to be sensitive to pollution, though resisting the physical and chemical effects of the waters tested (salinity and temperature, in particular). An evaluation was the made on the effects of these waters; first on larval production during a period of ten days, and then on ingestion by copepeds of a suspension of Pheodactylum tricormutum for four days.

The results obtained here with batches of sediment from the Marseilles region show that the larval production test is the most sensitive one. Indeed, the inhibition percentages found by the larval production test range from 35 to $100 \%$ (figure 1 ), whereas they vary from 5 to $55 \%$ with the algae consumption test (figure 2). With the larval production test, it is possible to classify sediments according to their ecological quality.

From this test, moreover, the presence of harmful substances in the sediments can be rapidly detected. Being reproductible and not expensive, it supplements the far too restrictive traditional chemical analyses. When applied to estuarine sedimentary zones, combined with other tests, it should help establish a quality coefficient for sediments based on experimentation.

Key-words : toxicology, pollution of sediment, copepods, larval production, consumption of algae.

\section{1 - INTRODUCTION}

Une grande part des polluants déversés dans les eaux se retrouve dans les sédiments ; aussi a-t-on pu dire que ces derniers se comportent comme des indicateurs de pollution à mémoire, témoins du degré de pollution (LESOUEF et al., 1979). Les sédiments constituent donc un biotope privilégié pour rechercher l'état de pollution d'un écosystème aquatique (MEARNS et al., 1986). 
L'analyse chimicue des polluants dans les sédiments est, certes, un moyen $d^{\prime}$ investigation performant pour détecter les zones de dégradation de la qualité des eaux, toutefois, de nombreux polluants échappent inévitablement au dosage. c'est, dans ce contexte, que des essais biologiques sont imperratifs ; sont alors pris en compte les phénomènes de synergie entre polluants, phénomènes que les analyses chimiques ne permettent pas de déceler. Sont pris en compte ếgalement les processus de photo et de biodégradation.

Les espèces cibles doivent répondre aux critères suivants d'expérimentation : cycle d'élevage complet bien maftrisé ; représentativité ; large répartition çéographique.

En outre, les tests seront simples et rapides de manière à ce qu'ils puissent être effectués en routine. Ils dojvent être également peu couteux, fiables, sensibles et réalisables toute l'année. Pour des raisons pratiques, une miniaturisation est souhaitable. Un des objectifs de ces tests est d'établir rapidement "l'état đe santé" d'un secteux cótier ; s'ils fournissent des réponses anormales, des recherches approfondies doivent etre entreprises, notament en ce qui concerne l'analyse chimique, pour déterminex les causes des perturbations.

Ces tests sexont utilisables avec des milieux aussi chargés en substances minérales et organiques que le sont les eaux interstitielles et les eaux de lessivage des sédiments. C'est-a-dire que les espèces cibles utilisées doivent être résistantes aux variations physico-chimiques inhérentes à ces milieux (salinité, température, teneur en oxygène, $\mathrm{pH}$ ) ce qui semble exclure les organismes océaniques.

\section{2 - Matériel et méthodes}

\section{2-1 Choix de l'organisme test}

Plusieurs travaux relatifs a Tigriopus brevicomis Maller, copepode que $1^{\prime}$ on trouve eréquement dans les flaques d'eau littorales de la zone intertidale atlantique, ont permis de préciser les conditions d'elevage et d'utilisation en ecotoxicologie (FALCHIER et al, , 1982 ; IASSUS et al., 1984. LE DEAN et MARCAILLOU - IE BAUT, 1984) . Ce copépode répond à tous les critères a'experrimentation précisés plus haut. Les tests sublétaux que nous développons dans le présent travail seront axés sux les modifications de la production larvaire et du comportement alimentaire.

Des expériences poursulvies depuis le debut de l'année 1985 (LACAZE et DUCPEUX, 1987) nous indiquent $I^{\prime}$ intêrét des tests sublétaux portant sur le comportement alimentaire de ce copépode. L'ingestion d'une suspension de la diatomée Phaeodactylum tricormutum par une population de copepodes est alors mesurée pendant 96 heures en fonction du degré de pollution. On évalue la diminution, du taux de chlorophylle $\alpha$ en fonction du temps. 


\section{2-2 Mesure de la consommation d'algues}

Les copépodes utilisés proviennent d'un élevage entretenu à l'Institut océanographique et développé a partir d'une population fournie par le laboratoire "effets biologiques des nuisances" de I'IFREMER/NANTES (LE DEAN et MARCAILLOU - LE BAU, 1984).

On dépose successivement dans les flacons de $20 \mathrm{~mL}$ en verre à col vissé 30 femelles ovigères de Tigriopus brevicornis, des cellules de Phaeodactylum tricormutum obtenues après centrifugation $(5000 \mathrm{t} / \mathrm{min}$., $5 \mathrm{~min}$.$) de 10 \mathrm{~mL} d$ 'une suspension de cette algue en phase exponentielle de croissance, enfin, l'eau à tester ou une de ses dilutions. Dans le cas des témoins utilisation d'une eati de mer de référence (cf. paragraphe 4). Pour chaque eau à tester (ou dilution) et pour les témoins, 4 flacons sont utilisés.

Après 96 heures, les copepodes sont retenus sur un tamis en nylon (vide de maille de $60 \mu \mathrm{m}$ ) et on dose la chlorophyle a de la suspension d'algues récoltée sur filtre whatman GF/C (méthode de LORENZEN, 1967). L'expérience est réalisée à l'obscurité et à la température de $25^{\circ} \mathrm{C} \pm 1{ }^{\circ} \mathrm{C}$. Au temps zéro plusieurs fractions de $10 \mathrm{~mL}$ de la suspension de $P$. tricomutum sont filtrées et la chlorophylle a dosée ; on obtient ainsi la concentration initiale.

Pour la station de vaine une étude plus détaillée, destinée à déterminer le broutage en fonction du temps est réalisée. Chaque jour un des 4 flacons est prélevé et la concentration en chlorophylle a de la suspension d'algues restantes est mesurée. Dans les conditions experimentales la consommation moyenne pour les témolns en 96 heures est de $1200 \mu \mathrm{g}$ de chlorophylle $a$.

\section{2-3 Mesure de 1a production larvaire}

Un essai porte sur 5 concentrations exprimées en pourcentage de l'extrait $(15,30,50,70,100)$. On utilise par concentration 5 lots de 10 femelles ovigẻres de Tigriopus (sacs ovigẻres oranges correspondant à des oeufs à terme de développement). Chaque lot est déposé dans 1 cristallisoir en verre de $20 \mathrm{~mL}$ contenant $15 \mathrm{~mL}$ d'eau de mer. de référence. Ces récipients sont couverts pour éviter l'évaporation. Les essais sont conduits a l'obscurité. Temperature $22^{\circ} \mathrm{C} \pm 1{ }^{\circ} \mathrm{C}$.

On évalue la production larvaire (nauplii et copépodites) pour une période de 10 fours. Au début de la periode expérimentale introduction de $2 \mathrm{mg}$ de spirulines lyophilisées par cristallisoir.

En fin de test les organismes issus des 5 cristallisoirs correspondant à une même concentration sont concentrés sur un tamis de $60 \mu \mathrm{m}$ de vide de maille; on procède alors au comptage globale des larves dans une cuve de Dolfuss.

Dans les conditions expéximentales la production larvaire moyenne pour les témoins est de 476 larves en 10 jours soit 9,5 larves par femelle. 


\section{2-4 Obtention des eaux à étudier}

Les échantillons de sédiment sont pxélevés dans des sites ayant fait 1 'objet de travaux antérieurs (ARNOUX et $a l ., 1985$; BARNIER, 1984 ; MILLE et al., 1981 ; MILlE et al., 1983) et ont donné lieu alors à des analyses granulométriques. Nous avons utilisé 2 techniques de traitement des sédiments selon qu'il s'agissait de sable ou de vase.

\section{a) Cas du sable. Echantillons : Les Embiez, La Seyne, Côte Bleue}

Pour chacun des sites précités, plusieurs $\mathrm{dm}^{3}$ de sédiments sont prélevés, égouttês, homogénéisés, répartis par fractions dans des bocaux en verre et congelês rapidement. Décongélation juste avant la réalisation des tests.

Pour obtenir, dans ce cas, les eaux à étudier on procède par lessivage. On utilise : 1 volume de sable égoutté et 1 volume d'eau de mer filtrée au moment de l'utilisation (Whatman GF/C). Cette eau de référence est prêlevée au large de Roscoff et conservée plusieurs mois à 1 'obscurité et à $15^{\circ} \mathrm{C}$. Brassage $30 \mathrm{~min}$. (agitateux universel Bioblock). Décantation 3 heures. Centrifugation du surnageant (5 000 tours/min., 5 min.). Equilibration de l'eau ainsi obtenue avec l'air par bullage (30 min.). Mesure du pH (pH des différentes eaux testées : par ordre décroissant : La Seyne : 8,40 - Les Emblez : 8,15 - Eau de dilution : 8,03 - Cóte Bleue : 7,87 - Sédiment pollué de référence : 7,79 )

\section{b) Cas de la vase. Echantilion de Vaine (étang de Berre)}

Cette vase est constituée de 70 a 80 \% de fractions fines (inférieures à $63 \mu \mathrm{m}$ ). Prélèvements par benne Orange Peel (2 à 3 litres) et centrifugation à 6000 tours/min. Teneur en eau : 35 . Récupération et conservation par congélation de l'eau interstitielle. Décongélation juste avant la réalisation des tests. Aération $30 \mathrm{~min}$. (bullage). Mesure du $\mathrm{pH}: 8,44$.

\section{c) Sédiment pollué de référence}

Pollution de 100 grammes de sable sec provenant de l'estuaire de la Rance (station du Prieure) (LACAZE et al., 1977) par $1 \mathrm{~mL}$ d'un pestrole brut arabian 1 ight (BAL) etêté a $150^{\circ} \mathrm{C}$. Elimination par distillation des composants dont les points d'ébullition sont superieurs a $150^{\circ} \mathrm{C}$ ).

Le sédiment sec et pollué est étalé dans une grande borte de pétri puis déposé dans un réacteur photochimique. Celui-ci est équipê d'une batterie de 5 lampes OSRAM L 20 w70 emettant uniquement dans 1 'ultraviolet. De meme que pour le sprectre solaire il n'y a pas d'émission au-dessous de $300 \mathrm{~nm}$. Cette technique d'irradiation est decrite par VERDU et al., (1984) et DUCREUX et IACAZE (1987). Après 96 neures d'irradiation le sédiment pollué est txaité comme dans le cas du sable (cf. a)

\section{2-5 Analyse chimique}

Elle porte sur le sediment de vaine : sels nutritifs, méthọde spectrophotometrique ; metaux lourds, dosages polarographiques ; hydrocarbures totaux (infrarouge). Résultats (cf. tableau 1). 
Tableau 1.- Analyse chimique de l'eau interstitielle du sédiment vaine $(22 / 10 / 86)$.

Table 1. - Chemical analysis of interstitial water from the Vaine sediment $(22 / 10 / 86)$

\begin{tabular}{|c|c|c|}
\hline & SELS NUTRITIFS & \\
\hline \multirow{9}{*}{-} & Nitrates & $14,8 \mu \operatorname{atg}$. N.L..$^{-1}$ \\
\hline & Ammonium & $708 \mu$ atg.N.L. ${ }^{-1}$ \\
\hline & Ortho-phosphates & $80,8 \mu \operatorname{atg}$. N.L. ${ }^{-1}$ \\
\hline & CHIOROSITE & $12,3 \%$ \\
\hline & HYDROCARBURES TOTAUX & $11,3 \mathrm{mg} . \mathrm{L} .^{-1}$ \\
\hline & METAUX LOURDS & \\
\hline & Zinc & $23,7 \mathrm{jg} . \mathrm{L.}^{-1}$ \\
\hline & Plomb & $50,9 \mu \mathrm{g} . \mathrm{L} .^{-1}$ \\
\hline & Cadmium & Non décelable (1) \\
\hline
\end{tabular}

(1) Sevil de détection $=0,20 \mu \mathrm{g} \cdot \mathrm{L}^{-1}$

\section{3 - RÉSULTATS}

\section{3-1 Eau interstitielle du sédiment Vaine (Etang de Berre)}

On note des réductions de 90 \& de la production larvaire et de 35 \& de la consommation de $P$. tricomution par $T$. brevicornis après 96 heures de séjour dans de l'eau interstitielle (figures 1 et 2 ) ; les réductions sont respectivement de 68 et 6 dans le cas de dilutions de moitié.

L'êtude cinétique du broutage effectuée pour cette localité de Vaine nous indique que la diminution de consommation par rapport aux témoins est peu importante le premier jour ; par contre, les copépodes ne se nourrissent plus après 48 heures (figure 3 ).

\section{3-2 Eaux de lessivage des sédiments Embiez, La Seyne et Côte Bleue}

Les résultats portés sux les figures 1 et 2 indiquent des réductions de la production larvaire et de la consomnation de $P$. tricornutum par T. brevicomis par rapport aux témoins pour tous les sédiments étudiés.

Dans le cas de la consomation d'algues cette réduction varie de $19 \%$ (Les Embiez) à 29 (Cote Bleue) ; elle est donc du méme ordre pour tous les échantillons. Nous notons pour la station cóte Bleue une réduction de 29 ce qui est inattenđu puisque il s'agit là d'une localité en principe non concernée par la pollution; on retrouve ici, en fait, la même anomalie que pour le "test photosynthèse" pratiqué sur les mêmes sites (IACAZE, 1987). 


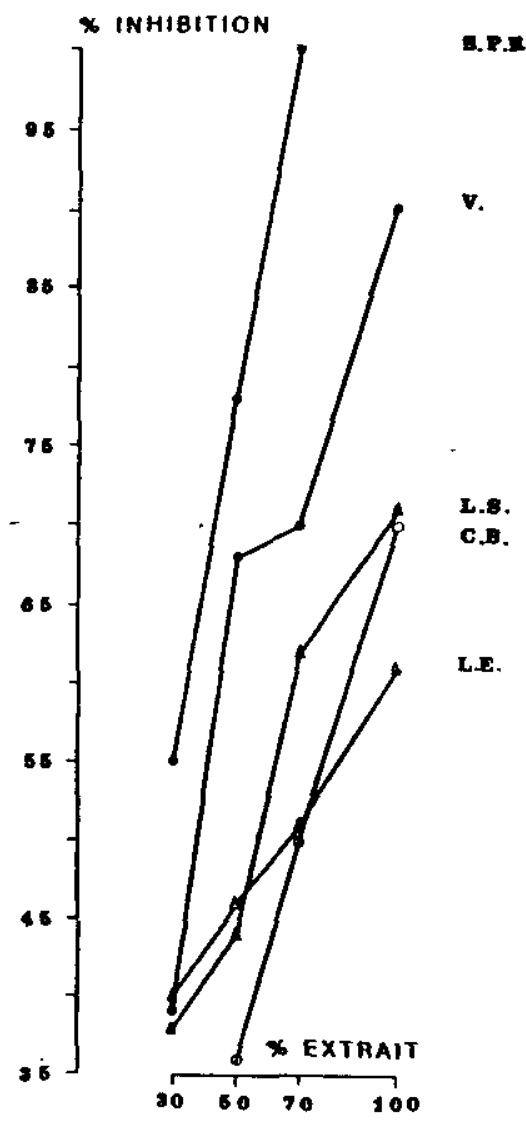

Figure 1.- Toxicités comparées dans le cas du test production larvaire. Les résultats sont exprimés en d'inhibition en fonction du degré de dilution.

Figure 1.- Comparative toxicities resulting from a larval production test. The results are expressed in \% of inhibition as a frotion of the degree of dilution.

S.F.R. : Sédiment pollué de référence; V. : Vaine; L.S. : La Seyne: C.B.: Côte Bleue; L.E. : Les Embiez.
S.P.R. : Reference polluted sediment ; V. : Vaine : L.S. : La Seyne : C.B. : Cote-Bleue; L.E. : Les Embiez. 


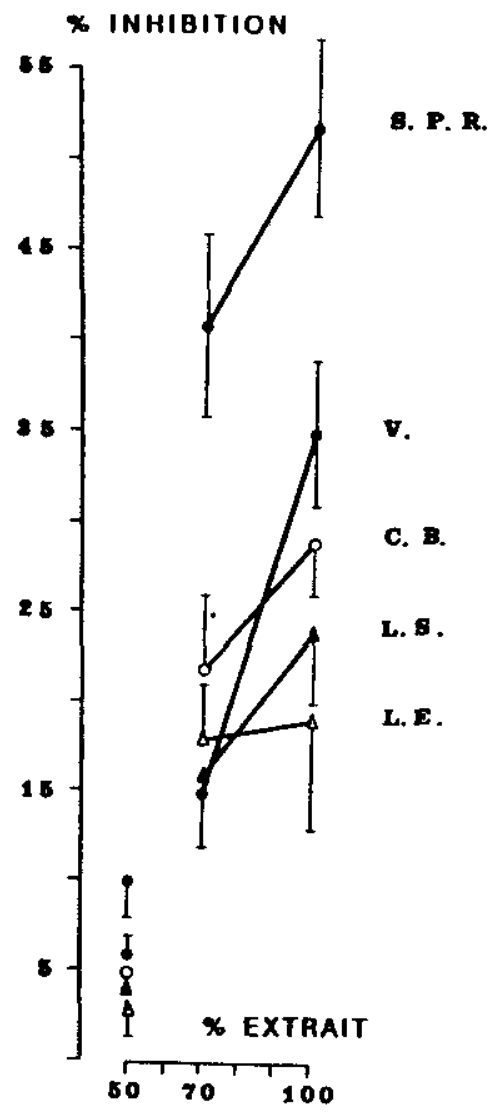

Figure 2.- Toxicités comparées dans le cas du test consommation d'algues. Les résultats sont exprimés en d'inhibition en fonction du degré de dilution. Les lignes verticales représentent l'intervalle de conflance pour $\mathrm{p}<0,05 ; \mathrm{n}=4$.

Figure 2.-Comparison of toxicities resulting from the algal test consumption. The results are expressed in $\%$ of inhibition as a function of the degree of dilution. Vertical lines represent the confidence interval for $p<0,05 ; n=4$.

S.P.R. : sédiment pollué de référence ; V. : Valne; L.S. : La Seyne ; C.B. : Côte Bleue ; L.E. : Les Embiez.
S.P.R. : Reference polluted sediment ; V. : Vaine; L.S. : La Seyne; C.B. : Côte-Bleue ; L.E. : Les Embiez. 


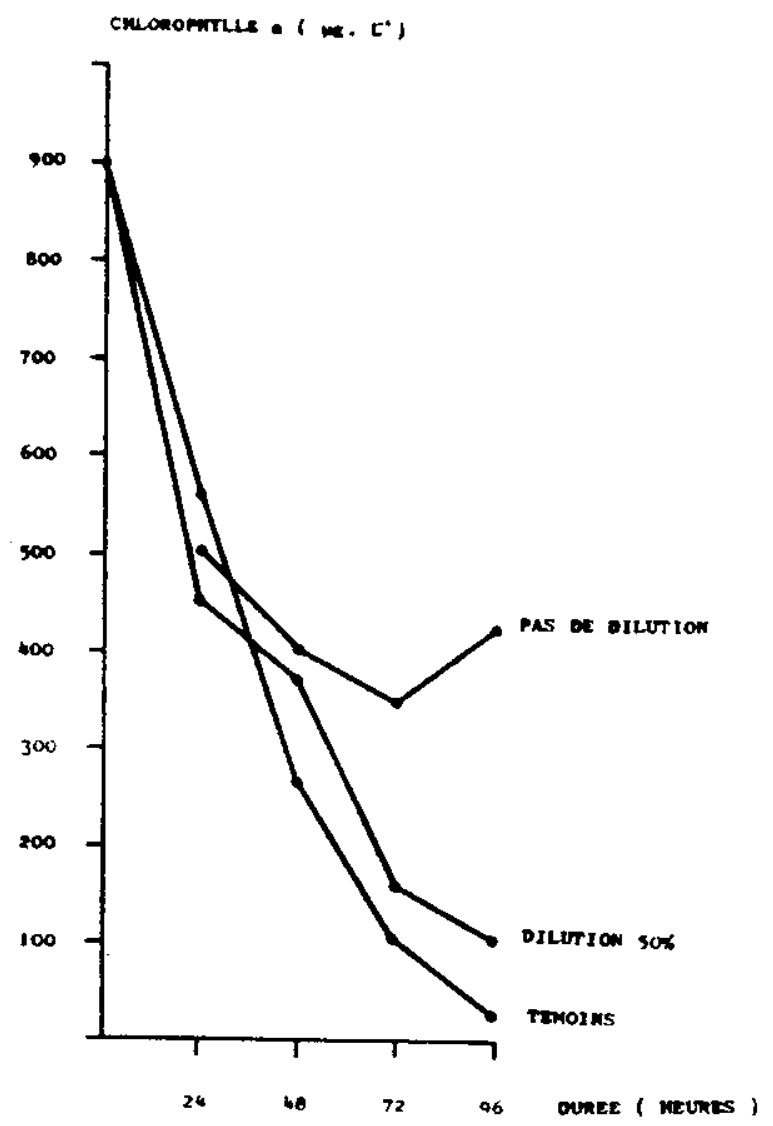

Figure 3.- Effets de l'eau interstitielle du séđiment vaine - (Etang de Berre) sur la consormation de P. tricornutum par $T$. brevicronis en fonctior du temps de contact. Les résultats sont exprimés en $\mu \mathrm{g} \cdot \mathrm{L}^{-1}$ de chlorophylle $a$.

Figure 3.- Effects of the interstitial water of sediment from Vaine (Etang de Berre) on the consumption of P. tricornutum by T. brevicornis as a function of time of contact. The results are expressed in $\mu g . L^{-1}$ of chlorophyll a.

La production larvaire présente par contre une réduction qui varie de 61 (Les Embiez) à 70 \& (La Seyne et Côte Bleue).

Le sédiment pollué de référence (pêtrole photooxydé), poux des dilutions de moitié, réduit de 78 la production larvaire et de 10 \& la consommation d'algues (figures 1 et 2 ). 
4 - Discussion

La cause des rêductions de la production larvaire et du broutage correspond à l'action simultanée de plusieurs polluants, certains étant déterminés par dosage, d'autres restant inconnus. A cette action s'ajoute celle des produits de dégradation. Dans la présente étude c'est donc l'action conjointe de l'ensemble de ces altéragènes qui est responsable des nuisances observés.

Si l'êvaluation de telles toxicités globales est d'un intérêt certain pour la surveillance de l'environnement, il est difficile, voire impossible de déterminer la cause exacte des effets observés. Néanmoins, les analyses chimiques réalisées pour l'eau interstitielle du sédiment de vaine nous permettent de fournir, pour cette station, quelques éléments d'explication.

Les données portées sur le tableau 1 nous indiquent des concentrations de 23,7 et $50,9 \mu \mathrm{g} \cdot \mathrm{L}^{-1}$ pour le zinc et le plomb. Récemment, lors d'une etude portant sur la toxicité de mélanges d'hydrocarbures aromatiques nous avons utilisé $\mathrm{SO}_{4} \mathrm{Zn} 7 \mathrm{H}_{2} \mathrm{O}$ comme polluant de référence (LACAZE et DUCREUX, 1987) ; les conditions expérimentales étant très proches de celles que nous utilisons ici. Pour $2 \mathrm{mg} \cdot \mathrm{L}^{-1}$ de $\mathrm{SO}_{4} \mathrm{Zn}$ l'alimentation est normale durant 24 heures; après ce laps de temps les copépodes ne se nourrissent plus. Avec $0,5 \mathrm{mg} \cdot \mathrm{L}^{-1}$ on note une diminution du broutage de 10 \& après 96 heures. Des données concernant la toxicité de ce polluant établies par le laboratoire des effets biologiques des nuisances de 1 'IFREMER (Nantes) a partir du même organisme et pour d'autres paramètres (tests letaux 96 heures, production de larves après 12 jours) nous permettent de comparer les sensibilités respectives des différents paramètres et nous fournissent des informations supplémentaires sur la toxiclté du zinc; ainsi pour $100 \mu \mathrm{g} \cdot \mathrm{L}^{-1} \mathrm{I}^{\prime}$ on décèle une légère diminution de la productior larvaire (LASSUS et al., 1984).

La concentration en zinc, considérée isolément n'est donc pas suffisante pour provoquer les effets décxits plus haut. Doit-on imputer les hydrocarbures?

Ies hydrocarbures étant dosés globalement, il est impossible de déterminer les composés responsables d'une éventuelle toxicité. Cela dit, la toxicité dépendra des types de composés présents et de l'état physicochimique des hydrocarbures (LACAZE, 1980). Une etude antérieure (IACAZE et DuCREuX, 1987) nous indique que $1,78 \mathrm{mg} \cdot \mathrm{L}^{-1}$ d'un mélange hydrosoluble d'hydrocarbures aromatiques légers et volatils (chaque composé étant déterminé et dosé) bloque totalement la consommation d'algues. Pour $1 \mathrm{mg} \cdot \mathrm{L}^{-1}$ l'alimentation partiellement inhibée pendant les premières 48 heures est ensuite complètement stoppée. Enfin, pour $0,35 \mathrm{mg} \cdot \mathrm{L}^{-1}$ on note une diminution de 27 \&u "broutage" après 96 heures. Toutefois, 1 'expérimentation nous indique qu'ici les hydrocarbures semblent se présenter sous forme de microémulsions - les composés volatils étant éliminés lors de "l'aération" - or, les copepodes harpacticoIdes sont généralement résistants aux hydrocarbures pétroliers présents sous cette forme (DATIA VENEZIA et FOSSATO, 1977), dans ce cas, il est habituel de noter que de fortes concentrations correspondent à de faibles toxicités. On ne doft dorc pas conclure hativement et déterminer un rôle pour les $11,3 \mathrm{mg} \cdot \mathrm{L}^{-1}$ d'hydrocarbures trouvés dans $\mathrm{l}^{\prime}$ eau interstitielle de vaine, il conviendrait de réaliser une analyse plus détaillée. 
Les résultats obtenus dans la présente étude nous indiquent une plus grande sensibilité cu test production larvaire qui, de ce fait, permet de mieux différencier les toxicités des eaux étudiées ; ainsi les pourcentages d'inhibition dans le cas du test production larvaire s'échelonnent de 35 à 100 \& (figure 1) aloxs qu'ils varient de 5 à 55 pour le test consomation d'algues (figure 2). Le test production larvaire nous permet donc de classer les sédiments selon leur qualité écologique ; on note ainsi par ordre des toxicités décroissantes : sédiment pollué de référence, Vaine, La Seyne et côte Bleue (pour ces 2 stations toxicitểs du meme orare), les Embiez.

Ce test permet de détecter de façon rapide la présence d'altéragènes dans les sédiments ; reprođuctible et peu coûteux, il complète avantageusement les analyses chimiques classiques beaucoup trop restrictives ; appliqué aux zones sédimentaires estuariennes, associé à d'autres tests dont certains réalisés avec des producteurs primaires (LACAZE, 1987) il devrait contribuer à établir un indice de qualité des sédiments basé sur 1 'expérimentation. Cet indice, allié aux indices dêjà existants (WILSON et al., 1987) pourrait apporter une plus grande précision pour l'évaluation de la qualité écologìque des estuaìres.

\section{REMERCIEMENTS}

Ce travail a été réalisé dans le cadre d'un contrat passé entre le ministère de I'Environnement et l'Institut océanographique (Laboratoire de Physiologie des Etres marins). Nous sommes redevables ã A. ARNouX et C. DIANA (Laboratoire d'Hydrologie, Faculté de Pharmacie, Marseille) qui ont effectué les prélèvements vaine (Etang de Berre) et les analyses chimiques. Nous remercions également G. MILLE (Centre de spectroscopie moléculaire, Faculté des Sciences et Techniques de Saint-Jérôme, Marseille) pour les autres prélèvements effectués sur la cóte méditerranéenne.

\section{RÉFÉRENCES BIBLIOGRAPHIQUES}

ARNOUX A., STORA G., DIANA C. (1986). In Bitu experimental study of the evaluation and recolonization of polluted sediments. Mar. Pollut. Bull., 16(B): 313-318.

BARNIER M. (1984). Evaluation des hydrocarbures dans les sédiments. Bassins experimentaux de biodegradation (Iles des Emblez) . D.E.A. d'Ecologie Mediterranénne.

DALLA VENEZIA L., FOSSATO V.U. (1977). Characteristics of suspensions of Kuwait oil and corexit 7664 and their short and long-term effects on Tisbe bulbisetosa (copepoda : Harpacticoida). Mar. Biol., 42 : $233-237$.

DUCREUX J., LACAZE J.C. (1987). Phytotoxicité des hydrocarbures aromatiques et des composés d'oxydation hydrosolubles après photooxydation d'un petrole brut. Sci. Eau, 6: 179-194.

FAICHIER M., IASSUS P., BARDOUIL M., LE DEAN L., TRUQUET P. , BOCQUENE G. (1982). Sensibilite thermique d'un copepode harpacticolde : Tigriopus brevicornis suller. Rev. trav. Inst. peches marit., $45(2): 141-153$. 
LACAZE J.C. (1980). La pollution pétrolière en milieu marin. De la toxicologie ¿ l'Ecologie. Masson ed., Ecologie appliquée et Sciences de l'environnement. $120 \mathrm{p}$. .

LACAZE J.C. (1987). Evaluation du degre de pollution des sediments marins au moyen d'un test subletal, 1 'effet sur l'activité photosynthétique de l'algue planctonique Phaeodactylum triconnutum mesuré par assimilation de ${ }^{14} \mathrm{C}$. Compt. Rend. Hebd. Seances Acad. Sci., paris, 305, série III : 515-520.

LACAZE J.C., VIEI.EDON DE NAİDE O., JOSEPH-GENEVET M., LE PEMP X. (1977). Influence de $l$ 'horizon de résuxgence sux la production primaire de deux plages du golfe de Saint-Malo. Ann. Inst, Ocecnogr. Paris, 53(2): 203-216.

LACAZE J.C., DUCREUX J. (1987). TOXicité des extraits aromatiques hydrosolubles issus de 2 petroles et d'une coupe petrolière. Effets sur l'activité photosynthetique de la diatomée Phaeodactylum triconnutum et sur l'ingestion de cette algue par le copépode Tigriopus brevicornis. Sci. Eau 6: 415-433.

LASSUS P., IE BAUT C., LE DEAN L., BARDOUIL M., TRUQUET P., BOCQUENE G. (1984). The use of harpacticoid copepods for testing the effects of chemicals on larval production. Ecotoxicological. testing for the mamine environment. PERSOONE G., JASPERS E., CLAUS C. eds. State Univ. Ghent and Inst. Mar. Scient. Res. Bredene, Belgium, 2: 131-142.

LE DEAN L., MARCAILLOU - IE BAUT $c$. (1984). Test d'Inhibition de développement d'un microcrustacé marin. Laboratoire effets biologiques des nuisances. IFREMER, Nantes, Rapport : 9 p..
LESOUEF A., BELAMIE R., MONTIEL A. (1979). Un indicateur de pollution mémoire. L'analyse des métaux dans les sédiments de rivière. J. Fr. Hydrol., $10(3)$ : 165-172.

LORENZEN C.J. (1967). Determination of chloxophyll and Pheoplgments : spectrometric equations, Limnol. Ocecnogr., 12: 343 .

MEAFNS A.J., SWARTZ R.C., CUMMINS J.M., DINNEL P.A., PLESHA P., CHAPMAN P.M. (1986). Inter-Laboratory Comparison of a sediment Toxlcity Test Using the Marine Amphipod, Rhepoxynius. Mar. Envir. Res., 19: 13-37.

MILIE G., DOU H., CRISTIANI G., GUISTI G. (1981). Hydrocarbures présents dans des sediments cotiers superficiels mediterraneens. I : Etude qualitative et quantitative fine. Environ. Pollut., serie $B, 2$ : $437-450$.

MTLLE G., GUTLIANO M., DOU H. (1983). Etude par spectroscopie de fluorescence u.v. des variations saisonnières du contenu en hyarocarbures aromatiques polycycliques de sediments cotiers superficiels mediterraneens (cote-Bleue, Bouche-du-phonel. Vie Marine, 5: 57-62.

VERDU J., THOMINETTE F. (1984). Photooxidative behaviour of crude oils relative to sea pollution. Part I : Comparative study of varlous crude oils and model systems. 91-104. Part II : Photoinduced phase separation. 105-115. Mar. chem., 15.

WILSON J.G. , DUCROTOY J.P., DESPREZ M. , ELKAIM B. (1987). Application d'indices de qualite écologique des estuaires en manche centrale et orientale. Compaxaison de la seine et de la Somme. Vie silieu, 37(1): 1-11: 